\title{
Repair Strategies for failed feature specification in Japanese: Evidence from loanwords, a reversing word game, and blending.
}

\author{
Daiho Kitaoka \\ University of Ottawa, Canada
}

\section{Problems and proposals}

This paper explores how place features of special moras in Japanese (long vowels, moraic nasals, double consonants) are specified when those features fail to be specified in a usual manner. In Japanese phonology, three types of particular segments are often distinguished from other 'plain' segments: moraic nasals, the second half of long vowels, and the first half of double consonants (cf. Kubozono 1999, Labrune 2012 for different views). I follow the traditional analyses in calling them special moras, and describe them as N, R, and Q, respectively. As shown in (1), place specification of special moras relies on neighbouring segments. (I employ '.' to indicate a mora boundary in examples.)

(1) Special moras
a. Long Vowel
ka.a.do /kaRdo/ 'card'
kju.u.ki /kyuRki/ 'inhalation'
b. Moraic Nasal
sa.m.ba /saNba/ 'samba'
ka.y.ki /kaNki/ 'ventilation'
c. Double Consonant
ni.k.ki /niQki/ 'dairy’

$$
\text { a.n.ta.i /aNtai/ 'stable' }
$$$$
\text { za.t.to /zaQto/ 'approximately' }
$$

The second half of a long vowel (R) as in (1a) has been argued to be a mora independent from the preceding CV mora. ${ }^{1}$ Coda consonants (nasals or obstruents) as in (1b) and (1c) undergo place assimilation with the following obstruents. A moraic nasal may also be realized as what I assume a placeless form, i.e., a uvular nasal $[\mathrm{N}]$ as in (2).

\section{(2) Placeless N: ka.N / $\mathrm{kaN} /$ 'can' te.N.i /teNi/ 'metastasis'}

Although the actual realization of the placeless $\mathrm{N}$ has been hotly debated, I assume that it is the least marked place of articulation in Japanese and that it surfaces by The Emergence of The Unmarked (TETU). In any case, this has no real bearing on my analysis, and for ease of exposition, I tentatively choose a uvular nasal $[\mathrm{N}]$ as the unmarked moraic nasal. (Note that ' $\mathrm{N}$ ' and ' $\mathrm{N}$ ' are distinguished in this paper: the former is meant to be an abbreviation of a moraic nasal; and the latter is a phonetic transcription of a uvular nasal.)

I demonstrate the cases where these regular processes of realizing special moras cannot be applied:

\footnotetext{
* My deepest thanks go to Marc Brunelle, Kevin McMullin, Sara Mackenzie, Phil Burness, Kathleen Strader, the language consultants, the audiences of the Aldridge Conference (2013), CUNY Phonology Forum (2014), CLA 2015 (Ottawa), WAFL 11 (York, 2015), MOTH Phonology Workshop 2016 (Ottawa), Seminar Series at Memorial University, Ottawa-Carleton Phonology Discussion Group, and the organizers, reviewers, and audience of the Annual Meeting on Phonology 4 (Los Angeles, 2016). I owe Sara Mackenzie the large portions of theoretical analysis on Sakasa Kotoba in this paper, which is largely based on Kitaoka and Mackenzie (2015, submitted). All errors are of course mine.

${ }^{1}$ Three patterns are identified for $\mathrm{CV}$ moras. In addition to $\mathrm{CV}, \mathrm{V}$ by itself may form a mora as in the first and fourth moras in a.n.ta.i (1b). The onset may consist of two consonants, in which case the second one should be [j] as in the first mora in kju.u.ki (1a). I describe these three $(\mathrm{CV}, \mathrm{V}$, and $\mathrm{CjV})$ as ' $\mathrm{CV}$ ' throughout this paper. The differences among them do not affect the discussion here or the outcomes of the word games.
} 
(recent) loanwords ${ }^{2}$, a reversing word game called Sakasa Kotoba, and blending. These processes often produce some marked outputs that are not commonly found in regular forms. I then discuss peripheral phonological processes in Japanese, and I propose three repair strategies in the cases of failed feature specification:

(3) Repair strategies of special moras

a. Over-generalization of regular structures found in core lexicon

(Yamato Japanese, Sino Japanese, entrenched loanword)

b. Irregular structures that are restricted to non-core lexicon

c. Game-specific structures

(Recent loanwords, interjections, onomatopoeia)

I illustrate that even in marked environments, repair processes make output structures as unmarked as possible by employing application of regular assimilation process (3a), or by realizing with realizing with TETU (3b). Based on the observations in the marked environments (mainly from Sakasa Kotoba), I further discuss the process of morification and underlying representations.

The proposed analyses contribute to the phonological study of Japanese (i) by describing peripheral phonological processes in Japanese; and (ii) by describing Sakasa Kotoba, a language game that has been hitherto paid little attention in the literature (e.g., Smith 1980), and that shows a novel language game type, i.e., total moraic reversal (see Bagemihl (1995) for the typology of language games).

This paper is organized as follows. After a brief description of Sakasa Kotoba and blending (Section 2), I illustrate the three repair processes in (3), based on the behaviours of special moras in marked environments (Sections 3 through 5). I then explore the theoretical implications in Section 6. Section 7 concludes the paper.

\section{Marked environments}

Some phonological processes force the special moras to appear at positions where they are not normally permitted by Japanese phonotactics and where the place feature of special moras are not specified in the typical ways as exemplified in (1) and (2). Recent loanwords often do not obey rules or constraints that core lexicon (Yamato Japanese, Sino Japanese, entrenched loanwords) are subject to (examples are provided in later sections). In this section, I provide brief descriptions of Sakasa Kotoba and blending, and show that these processes produce marked environments for the special moras.

Before presenting game data, comments on the data source is in order. The data of Sakasa Kotoba and blending presented in this paper is mainly based on the intuition of the author as a native Japanese speaker (Nagoya dialect). The data was also orally consulted informally with other native speakers (including Kyushu, Kansai, Kanto, and other Tokai dialects as well as Nagoya dialect).

2.1 Sakasa Kotoba Sakasa Kotoba is a language game used as a play mainly by kids. ${ }^{3}$ In Sakasa Kotoba, a game form is derived by reversing the moras; one could describe this as total moraic reversal. I also show that the game can be accounted for by referring to moras without referring to segments. Moreover, the game rules are not compatible with an analysis based on a prosodic constituent above the mora. In this section, I illustrate this by showing that the game does not target syllables. I begin with words that contain only CV moras:

\footnotetext{
${ }^{2}$ Unless otherwise noted, loanwords in this paper are meant to indicate recent loanwords. The Japanese lexicon includes three types of loanwords: (i) Sino Japanese, which came from the Chinese language(s) in various eras and regions, (ii) entrenched loanwords, which are mainly from English, and (iii) recent loanwords, which are Katakana descriptions of foreign words in any language. I consider the first two types to be included in core lexicon, and the third one to be genuine "loanwords." See Ito and Mester's series of work (e.g., 2008) with respect to lexical classes and their phonological phenomena.

${ }^{3}$ Be mindful not to confuse Sakasa Kotoba discussed here with other independent language games that are often called "Sakasa Kotoba" as well (e.g., Zuuja-go, Kaibun). Although these games are similar to Sakasa Kotoba in some aspects, the game rules or degree of orthographic influences are not the same as our Sakasa Kotoba. For the details of these games, see Ito, Kitagawa, and Mester (1996) for Zuuja-go, and Shimamura (1999) for Kaibun.
} 
(4) Sakasa Kotoba: CV

$\begin{array}{lll}\quad \text { Non-game form } & \text { Game form } & \text { Gloss } \\ \text { a. } \text { sa.ku.ra } & \text { rakusa / *arukas } & \text { 'cherry' } \\ \text { b. } \text { ka.o.ri } & \text { rioka /*iroak } & \text { 'fragrance' } \\ \text { c. } \text { kja.ku } & \text { kukja /*ukajk } & \text { 'guest' }\end{array}$

In the game form, $\mathrm{CV}$ moves around as a unit, which is traditionally analyzed as a mora. Although the game forms illustrate that the reversal does not target the segmental level ([*arukas, *iroak]), the forms in (4) are consistent both with a syllable-based analysis and with a mora-based analysis. When input words contain special moras, however, game forms suggest that it is moras that are reversed, since the special moras behave like $\mathrm{CV}$ moras. Consider the following:

(5) Sakasa Kotoba: $\mathrm{CV}+\mathrm{R} / \mathrm{N} / \mathrm{Q}$

\begin{tabular}{|c|c|c|c|c|}
\hline & \multicolumn{2}{|c|}{ Non-game form } & Game form & Gloss \\
\hline a. Long vowel (R) & ka.a.do & /kaRdo/ & $\begin{array}{l}\text { do.o.ka /doRka/ } \\
\text { cf. *dokaa, *doaka }\end{array}$ & 'card' \\
\hline b. Moraic nasal $(\mathrm{N})$ & ge.n.da.i & /geNdai/ & $\begin{array}{l}\text { i.da.y.ge /idaNge/ } \\
\text { cf. *idagen, *idange }\end{array}$ & 'present days' \\
\hline Double consonant $(\mathrm{Q})$ & ki.t.te & /kiQte/ & $\begin{array}{l}\text { te.k.ki /teQki/ } \\
\text { cf. *tekit, *tetki }\end{array}$ & 'stamp' \\
\hline
\end{tabular}

If the reversal targeted syllables, the game form would be dokaa in (5a), contrary to the attested dooka. The outputs of the game are created by totally reversing the order of moras, sustaining the mora-internal CV order. The game forms also demonstrate that the surface features of special moras are determined after reversal as the surface representations of special moras can be altered from the input form; e.g., from [a] to [o] in (5a). Thus, in the game form, the reversal occurs with a CV as well as each of R, Q, and N, as a unit.

Some might argue that Sakasa Kotoba is implemented orthographically rather than phonologically. This view makes sense since two of three writing systems in Japanese (Hiragana, Katakana) are largely mora based. Although I do not deny orthographic influence completely, I assume that Sakasa Kotoba is a phonological process at least when the input word is short enough (e.g., three moras). (For details and theoretical non-orthographic account of the game, see Kitaoka 2016, Kitaoka \& Mackenzie 2015, submitted).

The game rule, total moraic reversal, is exceptionless. Therefore, the reversing often produces marked sentences that are not found in core lexicon. I introduce such cases and analyses of them in the later sections.

2.2 Blending Blending is a phonological or morphological truncation process to compact a longer phrase(s). In particular, I focus on blending as in (6), wherein the first two moras in the first element and the last two moras in the second element are picked up and connected.

(6) Blending

Original forms

a. wa.n.da.a.fo.o.ge.ru /waNdaR foRgeru/

b. e.f.fe.ru. to.o

/eQferu toR/

Truncated forms
wa.y.ge.ru /waNgeru/
e.t.to.o /eQtoR/

Gloss

'Wandervogel' 'the Eiffel Tower'

In the same way as Sakasa Kotoba, the quality of the special moras is determined after truncation. For instance, $/ \mathrm{N} /[\mathrm{n}]$ in the input in (6a) becomes [n] in the output, assimilating to the place of following obstruent $[\mathrm{g}]$ in the output form. This truncation process also forces special moras to appear in marked environments, which I discuss in the next section. ${ }^{4}$ The optionality or bias to unmarked structure is found

\footnotetext{
${ }^{4}$ Since blending itself does not have such a strict rule that two moras each be extracted, speakers in this study needed to consciously pick up two moras each. I consider such conscious processes to be still valid even if there is some level of
} 
everywhere in the marked environments in the present paper, and is also characteristics of non-core lexicon and language games (Vaux 2011).

\section{Overgeneralization of regular structures}

In this and the following two sections, I introduce marked cases where place feature of the special moras are not specified in the usual manner as in (1) and (2), both of which are repeated here:

(7) Special moras
a. Long Vowel
ka.a.do /kaRdo/ 'card'
b. Moraic Nasal
sa.m.ba /saNba/ 'samba'
kju.u.ki /kyuRki/ 'inhalation'
ka.y.ki /kaNki/ 'ventilation'
c. Double Consonant
ni.k.ki /niQki/ 'dairy'
a.n.ta.i /aNtai/ 'stable'
za.t.to /zaQto/ 'approximately'
Placeless N: ka.N /kaN/ 'can' te.N.i /teNi/ 'metastasis'

(8)

The second half of a long vowel $\mathrm{R}$ is a copy of the first half (7a). The moraic nasal /N/ assimilates to the place of following obstruents (7b), or is realized with the placeless unmarked specification, i.e., a uvular nasal $[\mathrm{N}]$ when it comes the word-final position or it is followed by sonorants (including vowels) (8). The first half of a double consonant is also a copy of the following obstruent. In what follows, I demonstrate unusual processes of realization of the special moras as in (3), repeated here as (9).

(9) Repair strategies of special moras

a. Over-generalization of regular structures found in core lexicon

b. Irregular structures that are restricted to non-core lexicon

c. Game-specific structures

When the special moras fail to get place features in a usual manner, it employs regular structure (9a) similar to (7) (Section 3), or resorts to the unmarked structure (9b) similar to (8) (Section 4). I also show highly marked cases (9c), which can be found only in game forms (Section 5).

3.1 Loanwords In loanwords, voiced obstruents can be doubled as well as voiceless consonants as in (10), whereas only voiceless obstruents can be doubled in core lexicon.

(10) be.d.do /beQdo/ be.t.to /beQto/ 'bed' (Voiced obstruent)

$\mathrm{Q}$ in (10) copies the following voiced obstruent /d/ in the same way as that in core lexicon (7c). (Another possible way is to de-voice the voiced consonant as in be.t.to in order to fit the source word into Japanese native phonotactics. This alteration is not of interest in the present paper.)

Doubling is not limited to obstruents; sonorant consonants can also be doubled as in (11) (See below for vowels).

(11) a.r.ra.a /aQraR/ a.P.ra.a /aQraR/ 'Allah' (Sonorant)

This example also shows that $\mathrm{Q}$ in a marked environment employs a repair process wherein a regular structure is applied to the environment that is not found in core lexicon. (See the next section regarding another possible outcome, i.e., a glottal stop [?].)

3.2 Sakasa Kotoba The examples in (12) show that when a voiced obstruent or a sonorant precedes a (voiceless) double consonant in the input form in Sakasa Kotoba, the game rule, total moraic reversal, leads $\mathrm{Q}$ to precede the voiced obstruent.

conscious word-creation of the forms, since I assume that realization of special moras is still subconscious. It is beyond the focus of this paper how much speakers try to avoid marked structures, and how to truncate in blending. 
(12) Non-game form

a. ba.t.to /baQto/

b. ra.k.ka /raQka/

\section{Game form}

to.b.ba

ka.r.ra ka.?.ra /kaQra/
Gloss

'bat'

'fall'
(Voiced obstruent)

(Sonorant)

In (12), $\mathrm{Q}$ in the game forms copies the following voiced obstruent (12a) and the sonorant $/ \mathrm{r} /(12 \mathrm{~b})$, applying the regular doubling structure in (7c). (See the next section regarding another possible outcome in the case of sonorant, i.e., a glottal stop [?]). In case of nasals, the outcome is not as predicted (for some speakers):
(13) Non-game forms
Game forms
Gloss
me.k.ki /meQki/
ki.P.me, ??ki.m.me
'plate, gilding'
(Nasal)

Rather than simply copying the following nasal as in kimme, speakers resort to replace it with [?]. I assume that speakers avoid copying $[\mathrm{m}]$ in order to maintain recoverability of $\mathrm{Q}$ in the input word. In kimme created by employing the regular structure, the presence of the geminating mora is not apparent, since kimme is likely to be wrongly analyzed or perceived as /kiNme/.

3.3 Blending The over-application of regular process as in (7) is also found in blending. Consider the followings:

(14) Source forms

a. ka.t.to mo.de.ru /kaQto moderu/

b. ro.k.ku.fe.ra.a /roQkuferaR/

\section{Truncated forms}

ka.d.de.ru $\quad / \mathrm{kaQderu/}$

ro.r.ra.a ro.?.ra.a /roQraR/
Gloss

'cut model'

'Rockefeller'

In the same way as loanwords and the Sakasa Kotoba forms, Q in the truncated forms copies the following voiced obstruent (14a), or the sonorant $/ \mathrm{r} /(14 \mathrm{~b})$. (See the next section regarding another possible outcome in the case of sonorant, i.e., a glottal stop [?])

Thus, in the marked environments created in non-core lexicon (loanwords) or derived in game forms, the special mora Q may still employ the regular structure found in the core lexicon. Namely, Q doubles the following consonant in loanwords and game forms, whether it is a voiceless obstruent (which can be doubled in core lexicon as in (7)), or voiced consonants (whose doubled forms are not found in core lexicon).

\section{Irregular structures}

In addition to the over-application of regular structures as discussed in the previous section, failure to specify place feature of special moras can be repaired by creating marked structures. I illustrate that these irregular structures in loanwords can still be found in game forms, and hence not totally idiosyncratic to the game forms. I also demonstrate that this repair process is characterized by The Emergence of the Unmarked; special moras in marked environments are realized with the least unmarked specification, i.e., a uvular nasal [N] for N, and a glottal stop [?] for Q.

4.1 Loanwords As shown in (11), repeated below, Q preceding a sonorant either doubles the sonorant as in arraa or be realized as a glottal stop as in a?raa.

\section{(15) a.r.ra.a /aQraR/ a.P.ra.a /aQraR/ 'Allah' (Sonorant)}

The emergence of a glottal stop is not observed in core lexicon, but limited to non-core lexicon. Besides loanwords, a glottal stop is found in onomatopoeia (16a) and interjections (16b) and (16c).
(16) a. pe.?
$/ \mathrm{peQ} /$
(an onomatopoeia for spitting)
b. e.?
$/ \mathrm{eQ} / \quad$ (an interjection to express a surprise)
c. e.P...to.ne
/eQ...tone/ 'Let meeeeeee see.' ('...' indicates a pause)

Although a glottal stop is unlikely to be included in the phoneme inventory of Japanese phonology, I 
assume that it is the placeless (and hence unmarked) realization. I therefore consider the emergence of a glottal stop to be an instance of The Emergence of The Unmarked (TETU).

As discussed above, I claim that the use of a uvular nasal is also an instance of TETU. This is also found in core lexicon as discussed in (8), repeated below.

$$
\text { Placeless N: ka.N /kaN/ 'can' te.N.i /teNi/ 'metastasis' }
$$

When a moraic nasal does not have an obstruent to assimilate place to, it is realized as a uvular nasal, which is a placeless (unmarked) place specification. A uvular nasal is used in a marked environment as in (18), in which a moraic (and presumably also syllabic) nasal comes to the word-initial position.

(18) Word-initial moraic nasal (loanword)

n.dza.me.na $\sim$ N.dza.me.na /Njamena/ N'Djamena (foreign proper noun)'

In (18), $\mathrm{N}$ is realized either by assimilating place to the following obstruent as in ndzamena, or by resorting to the unmarked place specification, i.e., a uvular nasal, as in sdzamena. (I leave open for future research why $\mathrm{N}$ employs the placeless nasal even though there is an obstruent following which $\mathrm{N}$ can undergo place assimilation to.)

In fact, a word-initial moraic nasal appears in core lexicon in informal colloquial speech. However, the word-initial $\mathrm{N}$ in core lexicon appears to resist the placeless specification, and faithfully implements the regular assimilation process. Examine (19) (“-"' indicates morpheme boundaries).

(19) Word-initial moraic nasal (colloquial speech)
a. m-bjaku-neN
b. n.na ko.to
/N-byaku-neN/
/Nna koto/
how-hundred-year
such thing
'several hundred years'
'such thing'
cf. ${ }_{N}$-bjaku-neN
cf. * ${ }_{\text {Nna koto }}$

In informal colloquial speech, a syllable containing a moraic nasal often omits the CV. For instance, the word-initial $\mathrm{N}$ in (19a) was produced from $n a_{N} / \mathrm{naN} /$ 'what/how' by dropping the first mora /na/. One in (19b) was from sonna /soNna/ 'such.' In these cases, however, although $\mathrm{N}$ comes to the word-initial position, the nasal is not realized as a uvular nasal (cf. 18). With this contrast, I argue that the placeless specification is limited to marked environments in non-core lexicon (loanwords, interjections, onomatopoeia) and game forms (as discussed below). Thus, the use of $[\mathrm{N}]$ is an instance of TETU as a repair process in marked environments.

4.2 Sakasa Kotoba The same repair processes with TETU discussed above are employed in Sakasa Kotoba as well. As shown in (12b), repeated below, a sonorant in the game form may be either doubled as in karra (as discussed in Section 3), or replaced with a glottal stop as in ka?ra.
(20) Non-game form
Game form
Gloss
ra.k.ka /raQka/
ka.r.ra $\sim$ ka.r.ra /kaQra/ 'fall'
(Sonorant)

In the same way as loanwords in (15), Q preceding a non-obstruent (sonorant in (20)) may resort to the unmarked (i.e., placeless) specification. This replacement is also observed when a vowel follows $\mathrm{Q}$ in the game form:
(21) Non-game form
Game form
Gloss
a.k.ka /aQka/
ka.P.a $/ \mathrm{kaQa} /$
'deterioration'
(Vowel)

In (21), Q has no other option than resorting to the unmarked specification, i.e., a glottal stop, as no consonant follows it.

The example in (22) shows that the same goes with a moraic nasal. 
(22) Non-game form ki.ka.N /kikaN/
Game form

y.ka.ki N.ka.ki
/Nkaki/

Thus, the optionality observed in a loanword as in (18) (but not in core lexicon as in (19)) is also observed in Sakasa Kotoba; the word-initial $\mathrm{N}$ in the output form can be realized with the unmarked (placeless) specification as in skaki. Note that the use of a uvular nasal in the output in (22) cannot be accounted for by positing the surface form in the input is carried over in certain marked environment. Consider (23) first.

(23) Geimu-de-wa kasey-ga nani ni naru no?

game-in-тоPIC underline-NOM what to become QUESTION

'In the game, what will kasen become?'

Since Japanese has overt case markers, the input form is often attached to a case marker. As a result, the input word can undergo further assimilation; in (23), $/ \mathrm{N} /$ in kase $_{\mathrm{N}}$ assimilates place to the following obstruent $/ \mathrm{g} /$, which is the onset consonant of the nominative case marker -ga. As shown in (24), possible answers to the question in (23) do not include the surface form in the input ([n]):

(24) N.se.ka, n.se.ka, * „.se.ka /Nseka/

In (24), [ $\mathrm{y}]$ that is carried over from the input form in (23) is not acceptable. The word-initial nasal should either assimilate or be realized with the unmarked nasal. Thus, the use of a uvular nasal should be attributed to the environment, rather than access to the surface form. (See Section 5 for a different behaviour of R, which seems to carry over feature in the input.)

4.3 Blending As expected, the truncated forms in blending show the same behaviours of $\mathrm{Q}$ and $\mathrm{N}$ as those in loanwords and the Sakasa Kotoba forms. Examine (25) first.

(25) Source forms
a. ro.k.ku.fe.ra.a /roQkuferaR/
b. e.k.ku.su fa.i.ru /eQkusu fairu/
ro.r.ra.a ro.P.ra.a $/ \mathrm{roQraR/}$
e.P.i.ru leQiru/
'Rockefeller'
'X File'

Truncated forms

Gloss

When $\mathrm{Q}$ precedes a sonorant $/ \mathrm{r} /$, it is realized either by doubling it or by being replaced with a glottal stop as in (25a) (cf. (15) and (20) for the same realizations in loanwords and Sakasa Kotoba, respectively). A vowel following $\mathrm{Q}$ in the truncated form requires it to resort to the unmarked specification as in ePiru (cf. (21) for Sakasa Kotoba). The example in (26) shows that the emergence of the unmarked (placeless) specification is found in a moraic nasal as well.

(26) Source forms

pe.n ra.i.to /peN raito/
Truncated forms

pe.s.i.to /peNito/
Gloss

'pen light'

Thus, the repair processes may also involve irregular structures. Though irregular, the structures discussed thus far are instances of TETU, resorting to a placeless specification, i.e., [N] for a moraic nasal and [?] for the first half of a double consonant. The repair process appears to make output forms as unmarked as possible. On the other hand, these processes are specific to the repair processes; these are not idiosyncratic to the games, but not found in core lexicon, either.

\section{Game-specific structures}

Lastly, I introduce the third type of repair process, to be precise, cases of failure to repair in reasonable way (as discussed thus far), and employing game-specific structures ${ }^{5}$. Examine (27) first.

\footnotetext{
${ }^{5}$ For ease of exposition, marked structures derived from blending are also included in the 'game-specific' structures.
} 
(27) Non-game forms

a. ka.a $\quad / \mathrm{kaR} /$

b. ka.re.e /kareR/

\begin{tabular}{|c|c|}
\hline Game forms & \\
\hline $\begin{array}{l}\text { a.ka } \\
\text { ere } k a\end{array}$ & $\begin{array}{l}\text { /Rka/? } \\
\text { /Rreka/? }\end{array}$ \\
\hline
\end{tabular}

When the input form of Sakasa Kotoba ends with a long vowel as in (25), the strict application of the game rule produces the highly marked configuration in the output, wherein $\mathrm{R}$ comes to the word-initial position. In such cases, $R$ appears to carry over the place features of the non-game forms. In these cases, the repair mechanism fails to both apply regular structures found in core lexicon (discussed in Section 3), and employ irregular structures through TETU (discussed in section 4). This sounds natural if we assume that there is no unmarked vowel in Japanese. Namely, because TETU does not work in such cases, the output forms merely obey the faithfulness constraint, rather than deleting the vowel, inserting a vowel before it, or replacing it with something else.

One note on the unmarked specification is in order, however. One informant systematically inserts $/ \mathrm{u} /$ for these marked cases: /uka/ for (27a) and /ureka/ for (27b). This might suggest that / $\mathrm{u} /$ is the unmarked vowel (if ever there is marked/unmarked distinction in vowels in Japanese). The unmarkedness of $/ \mathrm{u} / \mathrm{might}$ be supported by the fact that $/ \mathrm{u} /$ is commonly used to fit loanwords into the CV mora formats in Japanese as in (28), in which a vowel $/ \mathrm{u} /$ is inserted after each consonant that is not followed by a vowel. ${ }^{6}$

(28) English club $\rightarrow$ Japanese kurabu

Another highly-marked game-specific structure involves R and N. Besides the case in (27), wherein R comes to the word-initial position, peculiar behaviours of special moras are also demonstrated in (29) and (30), wherein $\mathrm{R}$ follows a moraic nasal $\mathrm{N}$ in the output forms.

(29) Non-game forms ko.o.n.su.u.pu /koRN suRpu/

(30) Source words sa.n.ta ku.ro.o.su /saNta kuroRsu/
Game forms

pu.u.su.N.N.ko $\sim$ pu.u.su.y.y.ko

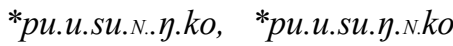
/puRsu NRko/
Gloss

'corn soup'

\section{Truncated form \\ Gloss \\ sa.N.s.su $\sim$ sa.n.n.su *sa.s.n.su, *sa.n.N.su 'Santa Claus' /saNRsu/}

In either case, $\mathrm{R}$ that in the input copies the preceding vowel copies the preceding moraic nasal in the output forms. The nasal is realized either with the unmarked specification $[\mathrm{N}]$ or with a regular assimilation structure as in puusuygko and sannsu. These specifications and the optionality are identical to what we have seen in the previous sections. Note, however, that the emergence of a uvular nasal in the output is not due to a position (as in (18)) or due to accessibility of the surface form (as in (27) for R). $\mathrm{N}$ in the game forms in (29) and (30) is not word-initial, and a uvular nasal is not used in the input, either.

Thus, the games may end up producing the game-specific structures, in which neither regular assimilation structures nor TETU works well. I have shown two idiosyncratic structures: (i) a word-initial $\mathrm{R}$ in the output carries over features in the input; and (ii) $\mathrm{R}$ copies $\mathrm{N}$, which either assimilates place to the obstruent following R, or resorts to the unmarked placeless specification [N]. Although I leave open the detail of derivation of the forms in (27), (29), and (30), I explore in the next section what these peculiar (but interesting) behaviours of special moras tell us about underlying representations and morification.

\section{Underlying representations and morification}

Based on the observations in the previous section, I explore the underlying forms and how moras are

\footnotetext{
${ }^{6}$ Loanwords adaptation also involves /o/ depending on environments, as in (i).

(i) English bottle $\rightarrow$ Japanese botoru.
} 
assigned. ${ }^{7}$ I argue that, contrary to some of the traditional views (including the present paper so far), the length of a vowel is morified underlyingly, as schematized in (31a). (For ease of exposition, I put a syllable on top of moras. The existence (or absence) of a syllable has no bearing on the discussion here.)

(31) a. Morification of a long vowel

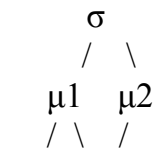

(C) $\mathrm{V}$ b. Morification of a moraic nasal and double consonant

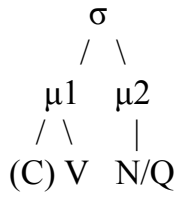

A long vowel is underlyingly associated with two moras as in (31a). The second mora ( $\mu 2)$ is R. On the other hand, a short vowel is associated with a single mora as in $\mu 1$ in (31b). The structure in (31a) is different from one in (31b) which is for a moraic nasal and double consonant. The latter structure has been posited in the literature to capture the independent moraic status of special moras including $\mathrm{R}$ as well as $\mathrm{Q}$ and N (e.g., Katada 1990). I deviate from Katada's structure in that a long vowel has a different morification process (Katada does not discuss morification, though).

First, I show the morification in the Sakasa Kotoba game forms:

(32) a. Non-game form: ka.a.do 'card'

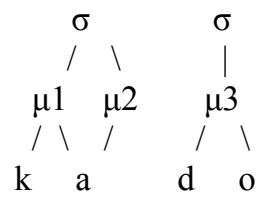

b. Reversal

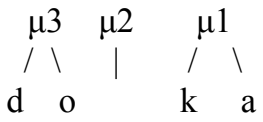

c. Game form: do.o.ka

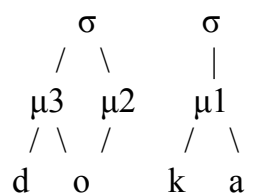

Suppose that total moraic reversal disassociates morification lines of vowels (32b). The empty mora, $\mu 2$ in (32b), is re-associated to the preceding vowel, [o] in (32c). Thus, the quality of a long vowel is altered in the output. The empty mora in (32b) is not re-associated with the following mora since a double consonant in the onset position violates a phonotactic rule in Japanese.

The structure in (31a) and the morification process in (32) explain the bizarre behaviours of a "long vowel" discussed in (29). First, examine the example with a shorter word in (33).

(33) Non-game form

ko.o.N

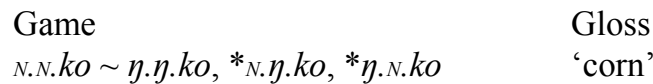

The moraic nasal preceding what was once the second half of a long vowel in the input, is realized with the unmarked place specification as in N.N.ko, or by assimilating to the obstruent following as in $\eta . \eta . k o$. In the

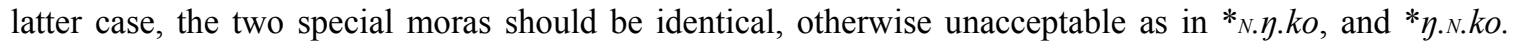
Note that a uvular nasal is emerged in the output not simply because $\mathrm{N}$ is word-initial, but rather because $\mathrm{N}$ precedes what was once the second half of a long vowel in the input (i.e., it gets syllabified), as illustrated

\footnotetext{
${ }^{7}$ The discussion in this section is largely based on Kitaoka (2016).
} 
in (29) and also as discussed in Section 4.

The structures in (34) show the step-by-step process of the game (i.e., total moraic reversal) and morification of the example in (33).

(34) a. Non-game form

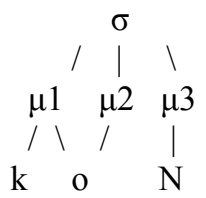

b. Reversal

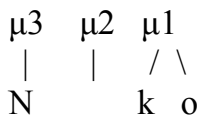

c. Game form

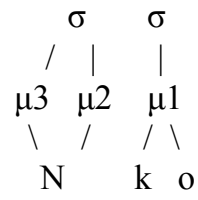

Following the mechanism in (32), a long vowel is associated to two moras in the input (34a). When the input word is reversed, the order of every mora is reversed and the second half of a long vowel ( $\mu 2)$ gets disconnected (34b). (Also, moras are de-syllabified.) The empty mora $(\mu 2)$ is re-associated with the preceding mora (34c). The first two moras in (34c) (both linked to N) are syllabified by themselves because they cannot be syllabified together with the following $/ \mathrm{ko} /$ due to a phonotactic rule. As discussed above, the syllabified $\mathrm{N}$ (typically in the word-initial position) has two options to be realized: (i) being specified with the unmarked place $[\mathrm{N}]$; or (ii) assimilating place to the following obstruent, if there is one. The two possible forms in (33) correspond these two options, respectively. It is obvious that the structure in (34c)

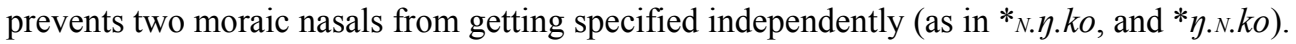

Thus, this section attempts to explore the underlying representation and morification of the second half of a long vowel (/R/ until the previous sections). The proposed structure and mechanism are consistent with the behaviour of a long vowel in the game, which would never be observed in regular forms in core lexicon.

\section{Conclusion}

I have demonstrated the repair strategies when place feature of the special moras in Japanese (the second half of a long vowel, moraic nasals, and the first half of a double consonant) fail to be specified in a usual manner. I posit three repair processes as in (35) based on the observations of marked environments (loanwords, Sakasa Kotoba, and blending).

(35) Repair strategies of special moras

a. Over-generalization of regular structures found in core lexicon

b. Irregular structures that are restricted to non-core lexicon, and that are instances of TETU

c. Game-specific structures

Lastly, I investigated a theoretical implication of the observation and analyses thus far, and argue that the vowel length is underlyingly distinct by the number of moras associated.

\section{References}

Bagemihl, Bruce. 1995. Language games and related areas. In John Goldsmith (ed.), The Handbook of Phonological Theory, 697-712. Cambridge, MA: Blackwell. 
Ito, Junko, and Armin Mester. 2008. Lexical classes in Phonology. In Shigeru Miyagawa and Mamoru Saito (eds.), The Oxford Handbook of Japanese Linguistics, 84-106. New York, NY: Oxford University Press.

Ito, Junko, Yoshihisa Kitagawa, and Armin Mester. 1996. Prosodic Faithfulness and Correspondence: Evidence from a Japanese argot. Journal of East Asian Linguistics 5(3): 217-294.

Katada, Fusa. 1990. On the representation of Moras: Evidence from a language game. Linguistic Inquiry 21(4): 641646.

Kitaoka, Daiho. 2016. Morification of special moras in Japanese: an analysis of a reversing language game. Unpublished paper. University of Ottawa, Canada.

Kitaoka, Daiho, and Sara Mackenzie. 2015. Evidence for the mora: analysis of a Japanese reversing game. Paper presented at the 11th Workshop on Altaic Formal Linguistics. University of York, UK. June 2015.

Kitaoka, Daiho, and Sara Mackenzie. Submitted. Moraic reversal and realization: Analysis of a Japanese language game.

Kubozono, Haruo. 1999. Mora and syllable. In Natsuko Tsujimura (ed.), The Handbook of Japanese Linguistics, 31-61. Malden, MA: Blackwell.

Labrune, Laurence. 2012. The Phonology of Japanese. New York, NY: Oxford University Press.

Shimamura, Keiichi. 1999. Sakasa Kotoba/Kaibun Asobi Daijiten [An encyclopedia for the play of Sakasa Kotoba/Kaibun. Tokyo: Tokyodo Shuppan.

Smith, Edward. 1980. Natural Phonology of Japanese. Doctoral Dissertation. University of Hawaii.

Vance, Timothy. 1987. An Introduction to Japanese Phonology. Albany, NY: State University of New York Press.

Vaux, Bert. 2011. Language games. In John Goldsmith, Jason Riggle, and Alan C. L. Yu (eds.), The Handbook of Phonological Theory (Third edition), 722-750. Malden, MA: Wiley-Blackwell. 